\title{
CARACTERÍSTICAS QUÍMICAS DO LIXIVIADO NA FASE DE ENRAIZAMENTO DE ESTACAS DE CACAU EM SUBSTRATOS ADUBADOS COM FÓSFORO ${ }^{(1)}$
}

\author{
José Olimpio de Souza Júnior ${ }^{(2)}$, Quirino Augusto de Camargo \\ Carmello $^{(3)}$ \& José Cláudio Faria ${ }^{(4)}$
}

\begin{abstract}
RESUMO
Para a produção de mudas em substratos, é intenso o uso de fertilizante e de irrigação, o que pode gerar perdas elevadas de nutrientes. O P é muito utilizado na preparação de substratos, mas estudos modelando suas perdas ainda são escassos. Este trabalho objetivou avaliar atributos químicos do lixiviado na fase de enraizamento de estacas de cacaueiro cultivadas sob irrigação intermitente em diferentes substratos e doses de $\mathbf{P}$. O delineamento experimental foi em blocos casualizados com três repetições, em esquema fatorial 5 x 5: cinco substratos (misturas, em volume, de 20, 35, 50, 65 e 80 \% de fibra de coco - FC, complementadas com Plantmax $\left.{ }^{\circledR}\right)$ e cinco doses de superfosfato triplo - SFT $\left(\mathrm{P}_{2} \mathrm{O}_{5}=0 ; 0,23 ; 0,46\right.$; 0,92 ; e $\left.1,84 \mathrm{~g} \mathrm{dm}^{-3}\right)$. A parcela foi formada por dois tubetes $\left(288 \mathrm{~cm}^{3}\right)$, com uma estaca herbácea de cacau por tubete. $O$ total do lixiviado de 28 dias foi coletado e medido, sendo a condutividade elétrica (CE) e o pH analisados em três amostras semanais. Os nutrientes $\mathrm{P}, \mathrm{Ca}, \mathrm{Mg}$ e $\mathrm{K}$ foram quantificados em amostras médias semanais. Os resultados foram submetidos à análise de regressão. Com o decorrer dos dias, o pH do lixiviado aumentou e a CE diminuiu, alcançando valores próximos aos da água de irrigação. O incremento de SFT diminuiu o pH e aumentou a CE do lixiviado. As variações de $\mathrm{pH}$ e CE foram mais rápidas e maiores quanto maior foi a proporção de $\mathrm{FC}$ no substrato. $\mathrm{O}$ teor e o conteúdo dos nutrientes no lixiviado decresceram rapidamente com o tempo, independente do substrato. O fornecimento de SFT aumentou a lixiviação de todos os nutrientes analisados. As perdas de $\mathrm{Ca}, \mathrm{Mg}$ e $\mathrm{K}$ foram proporcionais à riqueza desses nutrientes no substrato. A lixiviação do $\mathrm{P}$ aplicado variou de 39 a $74 \%$ e foi inversamente proporcional à capacidade de adsorção de fosfato do substrato.
\end{abstract}

Termos de indexação: lixiviação, perda de nutrientes, meio de cultivo, fibra de coco.

\footnotetext{
${ }^{(1)}$ Parte da Tese de Doutorado do primeiro autor, apresentada ao Curso de Pós-graduação em Solos e Nutrição de Plantas da Escola Superior de Agricultura "Luiz de Queiroz" - ESALQ/USP. Recebido para publicação em julho de 2007 e aprovado em abril de 2008.

(2) Professor do Departamento de Ciências Agrárias e Ambientais, Universidade Estadual de Santa Cruz - UESC. km 16, Rod. Ilhéus/Itabuna, CEP 45652-000 Ilhéus (BA). E-mail: olimpio@uesc.br

(3) Professor do Departamento de Ciência do Solo, Escola Superior de Agricultura "Luiz de Queiroz", Universidade de São Paulo - ESALQ/USP. Av. Pádua Dias 11, Caixa Postal 09, CEP 13418-900 Piracicaba (SP). E-mail: qaccarme@esalq.usp.br

(4) Professor do Departamento de Ciências Exatas e Tecnológicas, UESC. E-mail: jc_faria@uesc.br
} 


\title{
SUMMARY: CHEMICAL CHARACTERISTICS OF LEACHATE FROM PHOSPHORUS-FERTILIZED SUBSTRATES DURING ROOTING OF COCOA TREE CUTTINGS
}

\begin{abstract}
Fertilization and irrigation is intensively used for seedling production in substrate, which can result in high nutrient losses. Phosphorus $(P)$ is heavily used in the preparation of the potting mix, but studies on nutrient losses are scarce. The objective of this study was to evaluate the chemical attributes in leachate from potting mix during rooting of cocoa tree cuttings under intermittent irrigation and $P$ fertilization in a $5 \times 5$ factorial, complete randomized block design with three replications. The factors consisted of five substrates $\left(20,35,50,65\right.$ and $80 \%$ volume of coconut fiber $(C F)$ completed with Plantmax $\left.{ }^{\circledR}\right)$ and five triple superphosphate - TSP rates $\left(\mathrm{P}_{2} \mathrm{O}_{5}=0 ; 0.23 ; 0.46 ; 0.92\right.$ and $\left.1.84 \mathrm{~g} \mathrm{dm}^{-3}\right)$. The plot consisted of two tubes $\left(288 \mathrm{~cm}^{3}\right)$, with one herbaceous cocoa cutting per tube. Total leachate of 28 days was collected and measured, while electric conductivity (EC) and $p H$ were analyzed in three weekly samples. The nutrients $P, C a, M g$ and $K$ were quantified in weekly average samples and the data subjected to regression analysis. Over the course of time, the $\mathrm{pH}$ in the leachate increased and CE decreased. Increasing TSP doses reduced the $p H$ and increased the EC of the leachate. The higher the CF proportion, the faster and higher were $p H$ and $E C$ variations. Nutrients content and amount in leachate from all substrates tested decreased soon after starting the study. TSP supply increased leaching of all nutrients. $\mathrm{Ca}, \mathrm{Mg}$ and $\mathrm{K}$ losses were proportional to their availability in the substrate. Leaching of supplemented $P$ ranged from 39 to $74 \%$ and was inversely proportional to the phosphate adsorption capacity of the substrates.
\end{abstract}

Index terms: leaching, nutrient loss, growing media, coconut coir dust.

\section{INTRODUÇÃO}

Nos sistemas de produção de mudas em recipientes com substratos, em ambiente protegido ou semiprotegido, é intenso o uso de fertilizantes e de irrigação, sendo preocupantes as perdas de nutrientes, principalmente naqueles sistemas abertos onde a água de irrigação não recircula e é descartada. Essas perdas são muito elevadas para nutrientes considerados móveis, como o N (Thomas \& Perry Jr., 1980; Birrenkott et al., 2005), mas também podem ser bastante expressivas para aqueles considerados de baixa mobilidade no solo, como o P (Yeager \& Barrett, 1984; Ku \& Hershey, 1997; Godoy \& Cole, 2000).

Para garantir o sucesso na produção de mudas de cacau por estaquia, é indispensável que a estaca tenha folha remanescente madura, visando, principalmente, garantir o suprimento de carboidratos e nutrientes minerais móveis, e que a umidade do ar ao seu redor seja próxima de $100 \%$, para que não ocorra desidratação do tecido, queda das folhas remanescentes e subseqüente morte da estaca (Pyke, 1931; Evans, 1951; Murray, 1954). Em câmaras de enraizamento fechadas, é fácil manter a umidade relativa do ar elevada, mas em viveiros de produção de mudas, onde é rápida a perda de água por evaporação, faz-se necessário o uso de irrigação intermitente com alta freqüência, como avaliado por Ramos et al. (2006).

De modo geral, recomenda-se a utilização de fertilizantes fosfatados em adubação pré-plantio em substratos (Yeager \& Barrett, 1984; Williams \& Nelson, 1996; Bataglia \& Furlani, 2004). Essa prática, quando bem utilizada, favorece o desenvolvimento da planta (Godoy \& Cole, 2000), mas o uso de doses elevadas de $\mathrm{P}$, além de ser antieconômico, pode causar riscos ao ambiente, devido ao carreamento desse nutriente pela água drenada, principalmente quando se utilizam fertilizantes solúveis de liberação rápida (Broschat, 1995; Godoy \& Cole, 2000), elevada lâmina de água de irrigação (Ku \& Hershey, 1997), substratos com baixa capacidade de adsorção de P (Yeager \& Wright, 1982; Marconi \& Nelson, 1984; Williams \& Nelson, 1996) e plantas com taxas de enraizamento e de absorção de nutrientes lentas. Poder-se-ia pensar também que a macroporosidade alta de muitos substratos favoreceria a eluviação de partículas sólidas do fertilizante, fato não comprovado por Yeager \& Barrett (1984) para fertilizantes solúveis. Os autores, ao avaliar o $\mathrm{P}$ radioativo oriundo de lixiviado de substratos, concluíram que a lixiviação ocorreu apenas após a dissolução do superfosfato.

Estudos que avaliam as alterações químicas do lixiviado são importantes para a compreensão da dinâmica dos nutrientes no sistema, que, por sua vez, são subsídios para os estudos que visam à utilização racional dos fertilizantes e à minimização dos seus impactos no ambiente. Para a produção de mudas cítricas cultivadas em substrato, Bataglia et al. (2005) concluíram que o monitoramento da condutividade elétrica (CE) do lixiviado foi uma prática eficiente para 
evitar o suprimento excessivo ou deficiente de nutrientes. Birrenkott et al. (2005) afirmaram que é possível o uso de sistema de coleta de lixiviado para monitorar a disponibilização de nutrientes por fertilizantes de liberação controlada.

O objetivo deste trabalho foi avaliar as alterações no $\mathrm{pH}$ e na $\mathrm{CE}$ do lixiviado, bem como a lixiviação de $\mathrm{P}, \mathrm{K}, \mathrm{Ca}$ e $\mathrm{Mg}$ durante a fase de enraizamento de estacas, em sistema de produção de mudas de cacaueiro submetidas à irrigação intermitente e cultivadas em tubetes com substratos adubados com doses de superfosfato triplo.

\section{MATERIAL E MÉTODOS}

O experimento foi instalado em julho de 2005 em um viveiro de produção de mudas, com laterais e teto de tela plástica preta, com $50 \%$ de sombreamento, do Instituto Biofábrica de Cacau (IBC), no município de Ilhéus-BA.

O delineamento empregado foi o de blocos casualizados, com três repetições, em esquema fatorial 5 x 5: cinco substratos (misturas, em volume, de 20, $35,50,65$ e $80 \%$ de fibra de coco - FC, complementadas com Plantmax ${ }^{\circledR}$ - Pmax) e cinco doses de fósforo (0; 0,$23 ; 0,46 ; 0,92$ e $1,84 \mathrm{~g} \mathrm{dm}^{-3}$ de $\mathrm{P}_{2} \mathrm{O}_{5}$, na forma de superfosfato triplo moído) aplicadas no preparo dos substratos para o plantio das estacas de cacau. O fertilizante tinha $43,7 \%$ de $\mathrm{P}_{2} \mathrm{O}_{5}$, solúvel em CNA + água, $16,4 \%$ de $\mathrm{Ca}$, e 2,0 \% de $\mathrm{S}$.

A parcela foi formada por dois tubetes, com uma estaca herbácea de cacau por tubete, este em forma de cone, com as seguintes dimensões: $19 \mathrm{~cm}$ de comprimento, $5 \mathrm{~cm}$ de diâmetro na parte superior e capacidade para $288 \mathrm{~cm}^{3}$.

O Plantmax ${ }^{\circledR}$ (Pmax) utilizado foi o "florestal estaca", que é um substrato comercial composto de casca de pinus compostada, vermiculita expandida, carvão granulado e turfa. A FC utilizada constituiuse de um subproduto industrial do processamento do coco seco, formado por pó e fibras curtas do mesocarpo (casca, sem a epiderme) do fruto.

Os dois substratos originais, FC e Pmax, foram peneirados $(5 \mathrm{~mm})$ visando eliminar as partículas grosseiras, sendo esses substratos e suas misturas analisados quanto à densidade (Brasil, 2006) e às seguintes características químicas: $\mathrm{pH}$, condutividade elétrica (CE) e nutrientes, método $\mathrm{H}_{2} \mathrm{O} 1: 1,5 \mathrm{v} / \mathrm{v}$ (Sonneveld et al., 1974); capacidade de troca de cátions (CTC) (Brasil, 2006) e P remanescente (P-rem), adaptado de Alvarez V. et al. (2000), que foi o teor de $\mathrm{P}$ da solução de equilíbrio, após agitação por uma hora, de $10 \mathrm{~cm}^{3}$ de substrato com $100 \mathrm{~mL}$ de solução de $\mathrm{CaCl}_{2}$ 0,01 mol L-1, contendo $60 \mathrm{mg} \mathrm{L}^{-1}$ de $\mathrm{P}$, com repouso de cinco minutos e filtragem. Em amostra de substrato moída com pilão em almofariz, determinou-se o teor total de $\mathrm{C}$ e $\mathrm{N}$ em analisador elementar. Com os resultados do P-rem para os dois substratos originais, fez-se a curva de adsorção de $\mathrm{P}$ com doses sugeridas por Alvarez V. et al. (2000).

Utilizou-se sistema de irrigação do tipo nebulização intermitente automática, com água do rio Almada, programado para funcionar por $30 \mathrm{seg}$ a cada cinco minutos, no intervalo entre seis e 18 horas, sendo desligado nos períodos de chuva. Utilizaram-se bicos com vazão teórica de $40 \mathrm{~L} \mathrm{~h}^{-1}$, para pressão entre 250 e $300 \mathrm{kPa}$ e área irrigada de $4 \mathrm{~m}^{2}$ por bico. A vazão efetiva dos bicos foi medida duas vezes por semana.

Os coletores de lixiviado eram vidros de cor âmbar de capacidade para $1 \mathrm{~L}$ e foram instalados abaixo dos tubetes. Sacos plásticos vazados, presos à parte inferior dos tubetes, funcionaram como condutores do lixiviado até os coletores.

Durante 28 dias, foram coletadas três amostras semanais do lixiviado, com intervalo de dois ou três dias entre coletas. O lixiviado foi medido e nele determinaram-se o $\mathrm{pH}$ e a condutividade elétrica (CE). Alíquotas com $2 \%$ do volume coletado de cada parcela foram retiradas e acondicionadas em geladeira, até a formação das amostras compostas semanais, nas quais se determinaram: $\mathrm{P}$ (colorimetria), Ca e Mg (espectrometria de absorção atômica) e K (fotometria de chama). Nesse período, quatro amostras da água de irrigação também foram caracterizadas quimicamente.

Durante a dosagem de $\mathrm{P}$ no lixiviado, observou-se leitura de absorbância para os tratamentos que não receberam $\mathrm{P}$, a qual variou de acordo com o substrato e com a semana de amostragem. Essa leitura pode ser devida à presença de $\mathrm{P}$ nativo, como observado por Yeager \& Wright (1982), e, ou, presença de compostos orgânicos lixiviados dos substratos, que foram detectados pelo espectrofotômetro. Por isso, para o cálculo da concentração efetiva de P lixiviado, em relação ao aplicado, as leituras médias semanais dos tratamentos que receberam $\mathrm{P}$, para cada substrato e diluição, foram subtraídas daquelas que não receberam P.

Com os teores dos nutrientes e o volume do lixiviado semanal, calculou-se a quantidade total (conteúdo) de cada nutriente lixiviada semanalmente. O somatório desses valores semanais resultou no conteúdo acumulado lixiviado para cada nutriente.

Os valores de pH e CE do lixiviado das 12 coletas e os valores semanais dos teores, dos conteúdos e dos conteúdos acumulados de $\mathrm{P}, \mathrm{K}, \mathrm{Ca}$, e $\mathrm{Mg}$ foram submetidos à análise de variância e de regressão, sendo aceitos os modelos que apresentaram todos os coeficientes significativos até $10 \%$, pelo teste F, e o maior coeficiente de determinação ajustado. Para o $\mathrm{pH}$ e a CE, as regressões foram feitas para cada substrato, visando obter superfícies de resposta em função da dose de $\mathrm{P}$ e do tempo. Para os nutrientes, o substrato, expresso em porcentagem de FC, foi incluído no modelo, obtendo-se volumes de respostas. 


\section{RESULTADOS E DISCUSSÃO}

Durante o período experimental, a média das coletas da água de irrigação, oriunda do rio Almada, indicou: CE 0,088 $\mathrm{dS} \mathrm{m}^{-1}, \mathrm{pH}$ 7,34 e teores, $\mathrm{em} \mathrm{mg} \mathrm{L}^{-1}$, de 2,$1 ; 0,1 ; 0,9$ e $<0,1$ para $\mathrm{Ca}, \mathrm{Mg}, \mathrm{K}$ e $\mathrm{P}$, respectivamente. A irrigação média efetiva, calculada a partir dos volumes amostrados nas saídas dos bicos de irrigação, apontou lâmina diária em torno de $16 \mathrm{~mm}$, ou seja, 33 \% superior à lâmina teórica $(12 \mathrm{~mm})$. Isso ocorreu porque houve maior tempo e maior pressão de operação do sistema de irrigação. Houve também o aporte de água da chuva, que foi de 133,6 mm durante o período de 28 dias de coleta do lixiviado.

A CTC das misturas entre FC e Pmax, quando expressa por unidade de massa, variou pouco, apesar de o Pmax ter CTC 30 \% superior à da FC (Quadro 1). Isso ocorreu devido à grande diferença de densidade entre a FC e o Pmax, que foi respectivamente de 0,08 e $0,49 \mathrm{~kg} \mathrm{dm}^{-3}$ (Quadro 1). O valor da CTC de $450 \mathrm{mmol}_{\mathrm{c}} \mathrm{kg}^{-1}$ para a $\mathrm{FC}$ está dentro da faixa encontrada por Verhagen (1999) para 17 amostras de fibra de coco, que variou entre $347 \mathrm{e} 485 \mathrm{mmol}_{\mathrm{c}} \mathrm{kg}^{-1}$. Quando se expressou a CTC por unidade de volume, essa variação foi inversamente proporcional à porcentagem de FC no substrato (Figura 1), sendo a CTC do Pmax 752 \% superior à da FC.

$\mathrm{O}$ substrato Pmax foi mais rico em $\mathrm{S}$, Ca e $\mathrm{Mg}$, enquanto a FC apresentou maior teor de cátions monovalentes (K e Na) e de $\mathrm{P}$, além de ter relação $\mathrm{C} /$ $\mathrm{N}$ mais elevada (Quadro 1).

Outra característica química que merece atenção é o P-rem, pois é um bom indicativo da capacidade de adsorção de fosfato do material, entre outras aplicações (Alvarez V. et al., 2000). O P-rem aumentou linearmente com o aumento da proporção de FC no substrato (Figura 1), indicando que quanto maior a porcentagem de FC, menor será a adsorção de $\mathrm{P}$ pelo substrato. Esse raciocínio é ratificado quando se comparam as taxas de recuperação de $\mathrm{P}$ em solução, após 24 h de contato com os substratos, pois há adsorção de P pelo Pmax, enquanto a FC libera P para a solução, ou seja, ocorre dessorção (Figura 2), certamente porque o teor de P em solução na FC é alto (Quadro 1).

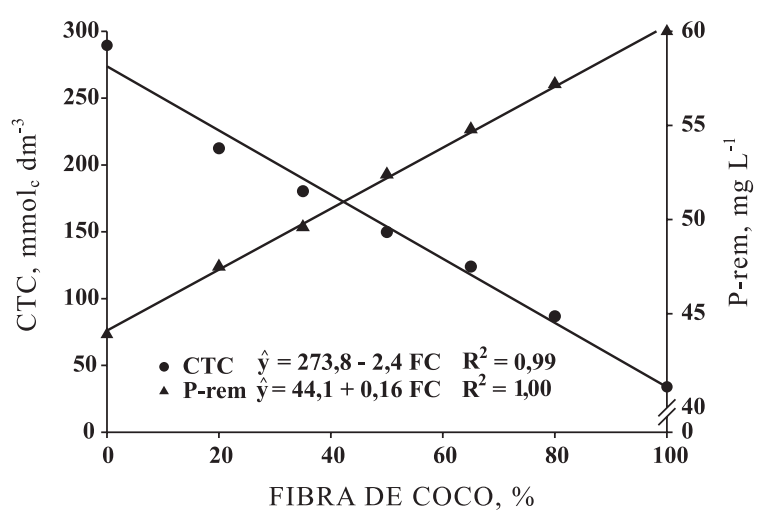

Figura 1. CTC e $P$ remanescente ( $P$-rem) em função da porcentagem de fibra de coco (FC) no substrato.

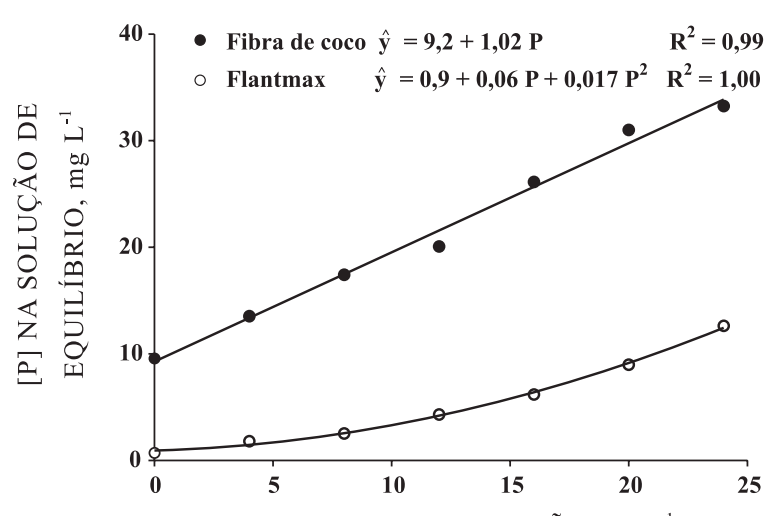

[P] INICIAL NA SOLUÇÃO, $\mathrm{mg} \mathrm{L}^{-1}$

Figura 2. Recuperação de $\mathrm{P}$, após $24 \mathrm{~h}$ de contato de 2,00 g dos substratos fibra de coco e Plantmax com $50 \mathrm{~mL}$ de solução com $P$.

Quadro 1. Análises ${ }^{(1)}$ dos substratos fibra de coco (FC), Plantmax (Pmax) e suas misturas (FC:Pmax)

\begin{tabular}{|c|c|c|c|c|c|c|c|c|c|c|c|c|c|}
\hline FC:Pmax & C & $\mathbf{N}$ & $\mathrm{C} / \mathrm{N}$ & CTC & DS & pH & $\mathbf{C E}$ & $\mathbf{P}$ & $\mathbf{S}$ & $\mathbf{K}$ & $\mathrm{Na}$ & $\mathrm{Ca}$ & $\mathbf{M g}$ \\
\hline$\%, \mathrm{v} / \mathrm{v}$ & \multicolumn{2}{|c|}{$-\mathrm{g} \mathrm{kg}^{-1}-$} & & $\mathrm{mmol}_{\mathrm{c}} \mathrm{kg}^{-1}$ & $\mathrm{~kg} \mathrm{dm}^{-3}$ & & $\mathrm{dS} \mathrm{m} \mathrm{m}^{-1}$ & 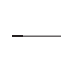 & 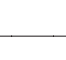 & $-\mathrm{m}$ & $\mathrm{L}^{-1}$ & & \\
\hline Pmax & 282 & 8,3 & 34 & 585 & 0,45 & 5,2 & 1,1 & 1,5 & 161 & 91 & 16 & 115 & 51 \\
\hline $20: 80$ & 307 & 8,0 & 38 & 530 & 0,36 & 5,2 & 1,1 & 1,6 & 156 & 113 & 59 & 105 & 48 \\
\hline $35: 65$ & 312 & 7,8 & 40 & 495 & 0,32 & 5,3 & 1,0 & 1,8 & 126 & 134 & 88 & 83 & 53 \\
\hline $50: 50$ & 318 & 7,7 & 41 & 505 & 0,26 & 5,3 & 0,9 & 1,6 & 99 & 150 & 127 & 58 & 42 \\
\hline $65: 35$ & 320 & 7,3 & 44 & 505 & 0,22 & 5,3 & 0,9 & 1,8 & 78 & 195 & 190 & 40 & 35 \\
\hline $80: 20$ & 325 & 6,6 & 49 & 495 & 0,15 & 5,3 & 0,8 & 2,5 & 43 & 205 & 206 & 15 & 20 \\
\hline $\mathrm{FC}$ & 451 & 3,7 & 121 & 450 & 0,06 & 5,6 & 0,8 & 7,9 & 2 & 288 & 265 & 1 & 4 \\
\hline
\end{tabular}

(1) Métodos: C e N totais, por analisador elementar; CTC e DS (densidade seca) (BRASIL, 2006) e pH, CE e elementos no extrato de $\mathrm{H}_{2} \mathrm{O}$ 1:1,5 v/v (Sonneveld et al., 1974). 
A isoterma de adsorção de $\mathrm{P}$ pelo Pmax é apresentada na figura 3 , onde se observa que a capacidade máxima de adsorção de $\mathrm{P}$ por esse substrato chega a aproximadamente $300 \mathrm{mg} \mathrm{kg}^{-1}$, considerada uma adsorção relativamente baixa quando comparada com a adsorção de P por solos (Andrade et al., 2002). Williams \& Nelson (1996) encontraram uma adsorção máxima de P para um substrato com $70 \%$ de turfa e $30 \%$ de perlita em torno de $250 \mathrm{mg} \mathrm{dm}^{-3}$.

Para todos os substratos, o $\mathrm{pH}$ do lixiviado apresentou um comportamento tipicamente quadrático para o $\mathrm{P}$ aplicado e o tempo, sendo a interação $\mathrm{P}$ x tempo também significativa (Quadro 2 e Figura 4a). Na verdade, todas as interações entre tempo, dose de $\mathrm{P}$ e tipo de substrato foram significativas para o $\mathrm{pH}$ do lixiviado.

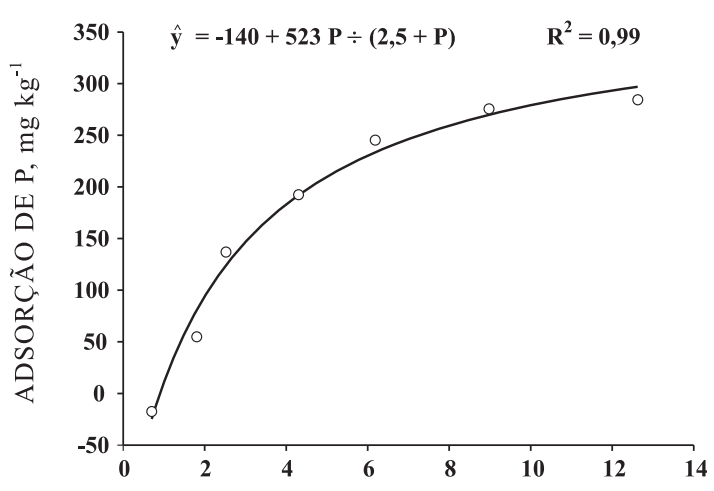

[P] NA SOLUÇÃO DE EQUILÍBRIO, $\mathrm{mg} \mathrm{L}^{-1}$

Figura 3. Adsorção de $P$ pelo substrato Plantmax em função da concentração de $P$ na solução de equilíbrio.
$\mathrm{O} \mathrm{pH}$ do lixiviado aumentou com o tempo (Figura 4a), alcançando valores próximos do $\mathrm{pH}$ da água de irrigação. A partir do $24^{\circ}$ dia, em média, iniciou tendência de queda, período próximo àquele apontado por Evans (1951) e Sacramento et al. (2001) como o início da produção das raízes, o que sugere possível início da absorção de nutrientes, com conseqüente alteração do $\mathrm{pH}$ do substrato e do seu lixiviado. Quanto maior a porcentagem de FC no substrato, mais rápida foi a alteração do $\mathrm{pH}$ do lixiviado (Figura 4a); para o substrato com $80 \%$ de FC e $20 \%$ de Pmax, esse equilíbrio foi atingido a partir do $14^{\circ}$ dia, evidenciando seu pequeno tamponamento.

$\mathrm{O}$ aumento da dose de $\mathrm{P}$, na forma de superfosfato triplo (SFT), diminuiu o valor do $\mathrm{pH}$ do lixiviado, sendo essa redução maior com o aumento da proporção de FC no substrato (Quadro 2). Certamente a acidez residual no SFT e, ou, o deslocamento de íons $\mathrm{H}^{+}$, para a solução do substrato, pelo $\mathrm{Ca}^{2+}$ do SFT, contribuíram para o abaixamento do $\mathrm{pH}$ do lixiviado. Porém, observa-se que a variação do $\mathrm{pH}$, apesar de significativa, foi de magnitude bem inferior, quando comparada com aquela provocada pelo tempo (Figura 4a).

Para a CE do lixiviado, as interações duplas entre substrato com o $\mathrm{P}$ e com o tempo foram significativas. Para três substratos, a interação $\mathrm{P} \times$ tempo também foi significativa (Quadro 2), evidenciando o efeito do fertilizante fosfatado em aumentar a CE do lixiviado, principalmente nos primeiro dias após sua aplicação e o início da lixiviação (Figura 4b).

Para todos os substratos, a CE decresceu rapidamente com o tempo (Quadro 2), indicando perda rápida de nutrientes, sendo essa queda pouco mais acentuada quanto maior a proporção de FC no

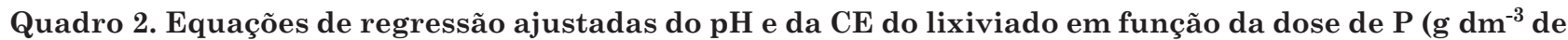
$\mathrm{P}_{2} \mathrm{O}_{5}$ ) e do tempo ( $\mathrm{T}$ - dia) e respectivos coeficientes de determinação, para cada substrato, misturas de fibra de coco (FC) e Plantmax (Pmax)

\section{FC:Pmax}

Equações de regressão
$\mathbf{R}^{2}$

$\mathrm{pH}$

\begin{tabular}{|c|c|c|}
\hline$\%, \mathrm{v} / \mathrm{v}$ & $\mathrm{pH}$ & \\
\hline $20: 80$ & $\hat{\mathrm{y}}=4,78-0,51^{* *} \mathrm{P}+0,05^{\circ} \mathrm{P}^{2}+0,19^{* *} \mathrm{~T}-0,0039^{* *} \mathrm{~T}^{2}+0,0109^{* *} \mathrm{PT}$ & 0,91 \\
\hline $35: 65$ & $\hat{\mathrm{y}}=4,65-0,71^{* *} \mathrm{P}+0,15^{\circ} \mathrm{P}^{2}+0,21^{* *} \mathrm{~T}-0,0044^{* *} \mathrm{~T}^{2}+0,0075^{\circ} \mathrm{PT}$ & 0,93 \\
\hline 50:50 & $\hat{\mathrm{y}}=4,72-0,81^{* *} \mathrm{P}+0,23^{* *} \mathrm{P}^{2}+0,23^{* *} \mathrm{~T}-0,0052^{* *} \mathrm{~T}^{2}+0,0153^{* *} \mathrm{PT}$ & 0,91 \\
\hline $65: 35$ & $\hat{\mathrm{y}}=4,48-0,84^{* *} \mathrm{P}+0,18^{* *} \mathrm{P}^{2}+0,28^{* *} \mathrm{~T}-0,0065^{* *} \mathrm{~T}^{2}+0,0162^{* *} \mathrm{PT}$ & 0,90 \\
\hline \multirow[t]{2}{*}{$80: 20$} & $\hat{\mathrm{y}}=4,51-0,85^{* *} \mathrm{P}+0,19^{* *} \mathrm{P}^{2}+0,31^{* *} \mathrm{~T}-0,0075^{* *} \mathrm{~T}^{2}+0,0134^{* *} \mathrm{PT}$ & 0,89 \\
\hline & $\mathrm{CE}\left(\mathrm{dS} \mathrm{m}{ }^{-1}\right)$ & \\
\hline $20: 80$ & $\hat{\mathrm{y}}=0,01+3,28^{* *} \mathrm{~T}^{-1}+0,71^{* *} \mathrm{P} \mathrm{T}^{-1}$ & 0,95 \\
\hline $35: 65$ & $\hat{\mathrm{y}}=-0,06+3,89^{* *} \mathrm{~T}^{-1}+0,26^{*} \mathrm{P} \mathrm{T}^{-1}$ & 0,96 \\
\hline $50: 50$ & $\hat{\mathrm{y}}=-0,06+3,48^{* *} \mathrm{~T}^{-1}$ & 0,93 \\
\hline $65: 35$ & $\hat{\mathrm{y}}=-0,16+5,23^{* *} \mathrm{~T}^{-1}$ & 0,98 \\
\hline $80: 20$ & $\hat{\mathrm{y}}=-0,15+3,94^{* *} \mathrm{~T}^{-1}+0,37^{* *} \mathrm{P} \mathrm{T}^{-1}$ & 0,96 \\
\hline
\end{tabular}


substrato (Figura 4b). Em média, a CE do lixiviado passou de 2,04 para $0,24 \mathrm{dS} \mathrm{m}^{-1}$ entre a primeira e a quinta coleta, ou seja, num intervalo de 12 dias, alcançando valor médio de $0,13 \mathrm{dS} \mathrm{m}^{-1}$ aos 21 dias após o início do experimento. Para os substratos mais ricos em Pmax, a CE do lixiviado alcançou valores levemente superiores aos da água de irrigação, e para aqueles mais ricos em $\mathrm{FC}$ a $\mathrm{CE}$ do lixiviado ficou semelhante ao da água de irrigação. Kerr \& Hanan
(1985) também observaram decréscimo rápido da CE do lixiviado de 10 substratos salinizados.

No quadro 3 são apresentadas as equações de regressão ajustadas (volumes de resposta) do teor, do conteúdo e do conteúdo acumulado de $\mathrm{P}, \mathrm{K}, \mathrm{Ca}$ e $\mathrm{Mg}$ no lixiviado em função da dose de $\mathrm{P}$, da porcentagem de FC e do tempo. Para facilitar a visualização da magnitude da lixiviação, são apresentadas, na figura 5, algumas superfícies de resposta oriundas de
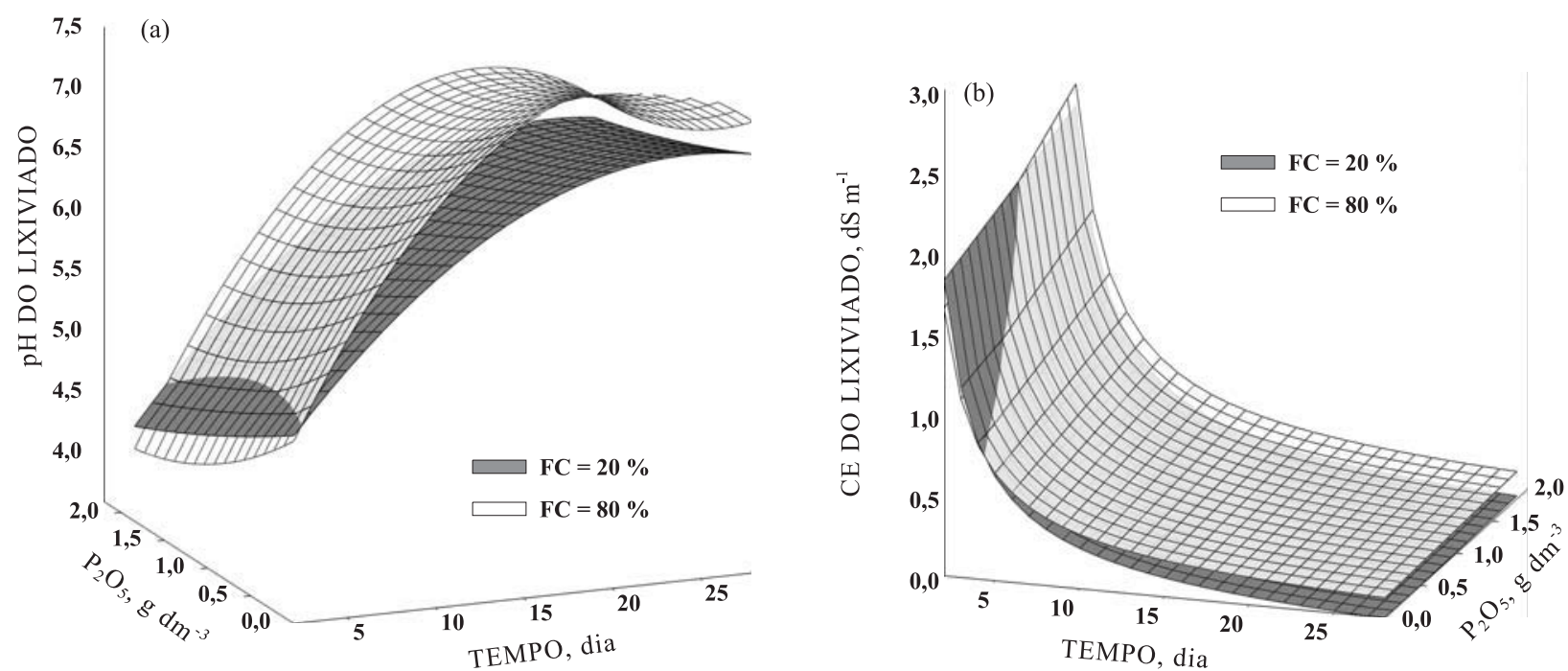

Figura 4. pH (a) e condutividade elétrica - CE (b) do lixiviado em função do tempo e da dose de $\mathrm{P}$, para os substratos com 20 e $80 \%$ de fibra de coco (FC).

Quadro 3. Equações de regressão ajustadas do teor, do conteúdo semanal ou do conteúdo acumulado de nutrientes no lixiviado em função da dose de $\mathrm{P}\left(\mathrm{g} \mathrm{dm}^{-3}\right.$ de $\left.\mathrm{P}_{2} \mathrm{O}_{5}\right)$, da porcentagem de fibra de coco no substrato (FC - \%) e do tempo ( $\mathrm{T}$ - dia) e respectivos coeficientes de determinação

\begin{tabular}{|c|c|c|}
\hline Nutrie nte & Equações de regressão & $\mathbf{R}^{2}$ \\
\hline \multicolumn{3}{|c|}{ Teor $\left(\mathrm{mg} \mathrm{L}^{-1}\right)$} \\
\hline $\mathrm{P}$ & $\hat{\mathrm{y}}=0,3-41^{*} \mathrm{~T}^{-1}-0,42^{* *} \mathrm{P} \mathrm{FC}+250^{* *} \mathrm{P} \mathrm{T}^{-1}+0,86^{* *} \mathrm{FC} \mathrm{T}^{-1}+6,3^{* *} \mathrm{P} \mathrm{FC} \mathrm{T}^{-1}$ & 0,95 \\
\hline $\mathrm{K}$ & $\hat{\mathrm{y}}=-20+846^{* *} \mathrm{~T}^{-1}-0,44^{* *} \mathrm{FC}+7,4^{* *} \mathrm{FC} \mathrm{T}^{-1}$ & 0,91 \\
\hline $\mathrm{Ca}$ & $\hat{\mathrm{y}}=-20-5,47^{* *} \mathrm{P}+0,16^{* *} \mathrm{FC}+536^{* *} \mathrm{~T}^{-1}+140^{* *} \mathrm{P} \mathrm{T}^{-1}-4,9^{* *} \mathrm{FC} \mathrm{T}^{\cdot 1}$ & 0,86 \\
\hline $\mathrm{Mg}$ & $\hat{\mathrm{y}}=-13-2,0^{* *} \mathrm{P}+0,07^{* *} \mathrm{FC}+368^{* *} \mathrm{~T}^{-1}+103^{* *} \mathrm{PT}^{-1}-2,6^{* *} \mathrm{FC} \mathrm{T}^{-1}-0,74^{* *} \mathrm{P} \mathrm{FC} \mathrm{T}^{-1}$ & 0,87 \\
\hline \multicolumn{3}{|c|}{ Conteúdo semanal (mg por tubete) } \\
\hline $\mathrm{P}$ & $\hat{\mathrm{y}}=0,1+6,5^{* *} \mathrm{P}-0,39^{* *} \mathrm{PFC}+78^{* *} \mathrm{P} \mathrm{T}^{-1}+6,3^{* *} \mathrm{P} \mathrm{FC} \mathrm{T}^{-1}$ & 0,97 \\
\hline $\mathrm{K}$ & $\hat{\mathrm{y}}=-4,0-4,0^{* *} \mathrm{P}-0,33^{* *} \mathrm{FC}+567^{* *} \mathrm{~T}^{-1}+3,6^{* *} \mathrm{FC} \mathrm{T}^{-1}+1,5^{* *} \mathrm{P} \mathrm{FC} \mathrm{T}^{-1}$ & 0,96 \\
\hline $\mathrm{Ca}$ & $\hat{\mathrm{y}}=-11-3,4^{* *} \mathrm{P}+0,08^{* *} \mathrm{FC}+346^{* *} \mathrm{~T}^{-1}+93^{* *} \mathrm{P} \mathrm{T}^{-1}-3,1^{* *} \mathrm{FC} \mathrm{T}^{-1}$ & 0,92 \\
\hline $\mathrm{Mg}$ & $\hat{\mathrm{y}}=-6,1+249^{* *} \mathrm{~T}^{-1}+32^{* *} \mathrm{P} \mathrm{T}^{-1}-1,8^{* *} \mathrm{FC} \mathrm{T}^{-1}$ & 0,90 \\
\hline \multicolumn{3}{|c|}{ Conteúdo acumulado (mg por tubete) } \\
\hline $\mathrm{P}$ & $\hat{\mathrm{y}}=0,2+58^{* *} \mathrm{P}+0,29^{* *} \mathrm{P} \mathrm{FC}-303^{* *} \mathrm{P} \mathrm{T}^{-1}+2,0^{* *} \mathrm{P} \mathrm{FC} \mathrm{T}^{-1}$ & 0,99 \\
\hline $\mathrm{K}$ & $\hat{\mathrm{y}}=165-0,22^{*} \mathrm{FC}-664^{* *} \mathrm{~T}^{-1}+0,17^{* *} \mathrm{P} \mathrm{FC}+3,7^{* *} \mathrm{FC} \mathrm{T}^{-1}$ & 0,83 \\
\hline $\mathrm{Ca}$ & $\hat{\mathrm{y}}=57+12^{* *} \mathrm{P}-0,56^{* *} \mathrm{FC}-57^{* *} \mathrm{~T}^{-1}+0,06^{*} \mathrm{P} \mathrm{FC}-33^{* *} \mathrm{P} \mathrm{T}^{-1}$ & 0,88 \\
\hline $\mathrm{Mg}$ & $\hat{\mathrm{y}}=48+9,1^{* *} \mathrm{P}-0,45^{* *} \mathrm{FC}-60^{* *} \mathrm{~T}^{-1}-56^{* *} \mathrm{P} \mathrm{T}^{-1}+0,55^{* *} \mathrm{P} \mathrm{FC} \mathrm{T}^{-1}$ & 0,85 \\
\hline
\end{tabular}

** e *, para p $<1 ; 5$ e $10 \%$ pelo teste $\mathrm{F}$, respectivamente. 
cortes das equações apresentadas no quadro 3 para o conteúdo lixiviado acumulado de cada nutriente.

O volume médio lixiviado foi de $124 \mathrm{~mL}$ por tubete por dia, e o coeficiente de variação médio de $26,6 \%$, sendo esse valor considerado elevado para um sistema de irrigação, que, segundo Siqueira \& Almeida (2005), apresenta coeficiente de uniformidade elevado, em torno de 97 \%. Essa variação do volume lixiviado pode ter ocorrido devido aos seguintes fatores: os substratos FC e Pmax apresentam capacidades de retenção e drenagem de água distintas (Siqueira \& Almeida, 2005); há possíveis efeitos de "guarda-chuva" e de "calha" das folhas remanescentes das estacas de cacau, que podem, respectivamente, reduzir a água efetiva que chega ao substrato, por efeito de cobertura parcial do tubete vizinho, ou aumentá-la, devido ao direcionamento da água captada pela folha para a base da estaca e conseqüentemente para o tubete; o sistema de coleta de lixiviado utilizado não é hermeticamente fechado, o que pode capturar alguma água não oriunda da lixiviação do tubete. Esses problemas são amenizados quando se considera o volume lixiviado, ou seja, utiliza-se o conteúdo em vez do teor. Isso justificaria os melhores ajustes, maiores coeficientes de determinação das equações de regressão para o conteúdo lixiviado dos nutrientes, em relação à sua concentração (Quadro 3).

(a)

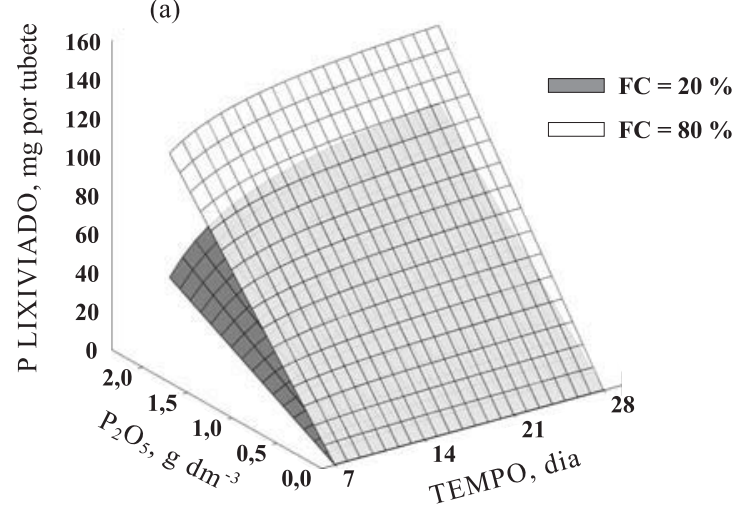

(c)

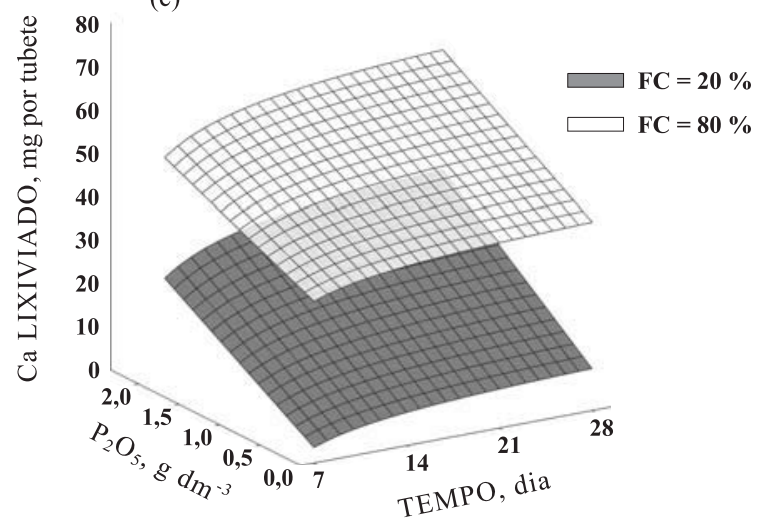

O tempo foi determinante na lixiviação dos nutrientes (Quadro 3), visto que os teores e os conteúdos dos nutrientes no lixiviado diminuíram rapidamente com o tempo, sendo as maiores perdas observadas na primeira semana e, obviamente, o conteúdo acumulado de nutrientes lixiviados aumentou com o tempo (Figura 5a,b,c,d). Os teores médios no lixiviado das quatro semanas analisadas foram respectivamente de: 41,$2 ; 4,2 ; 3,3$ e $2,3 \mathrm{mg} \mathrm{L}^{-1}$, para o $\mathrm{Ca} ; 31,0 ; 4,4 ; 3,2$ e 2,0 $\mathrm{mg} \mathrm{L}^{-1}$, para o $\mathrm{Mg}$ e 136,30 , 17 e $10 \mathrm{mg} \mathrm{L}^{-1}$, para o K.

Para o P, as perdas acumuladas até os 28 dias variaram de 39 a $74 \%$ do $\mathrm{P}$ aplicado, sendo que em média $63 \%$ dessa perda ocorreu na primeira semana. A lixiviação elevada de $\mathrm{P}$ em substratos formados por misturas de casca de pinus, turfa e areia também foi observada por Yeager \& Barrett (1984), que encontraram perdas médias acumuladas de 30, 55 e $71 \%$ dos $261 \mathrm{mg} \mathrm{dm}^{-3}$ do $\mathrm{P}$ aplicado, após um, sete e 21 dias, respectivamente.

A adição de SFT aumentou o conteúdo lixiviado de K, Ca e Mg (Quadro 3 e Figura 5b,c,d), apesar de o teor de $\mathrm{K}$ no lixiviado não ter sido significativamente afetado pelas doses desse fertilizante (Quadro 3), evidenciando novamente a superioridade do uso do conteúdo em relação ao teor. Certamente o Ca do STF

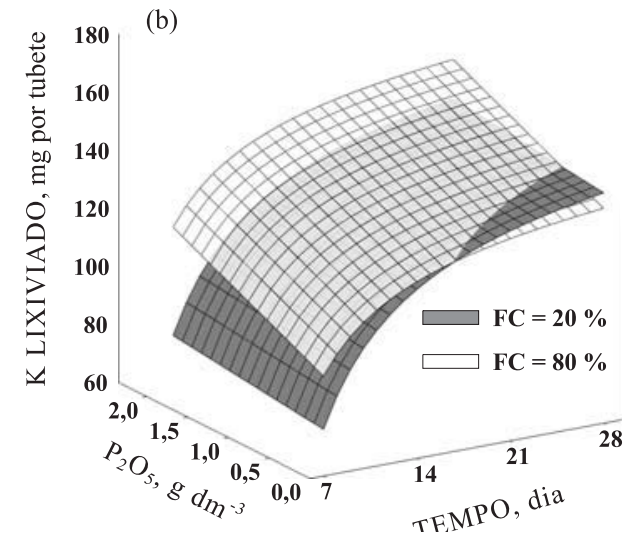

(d)

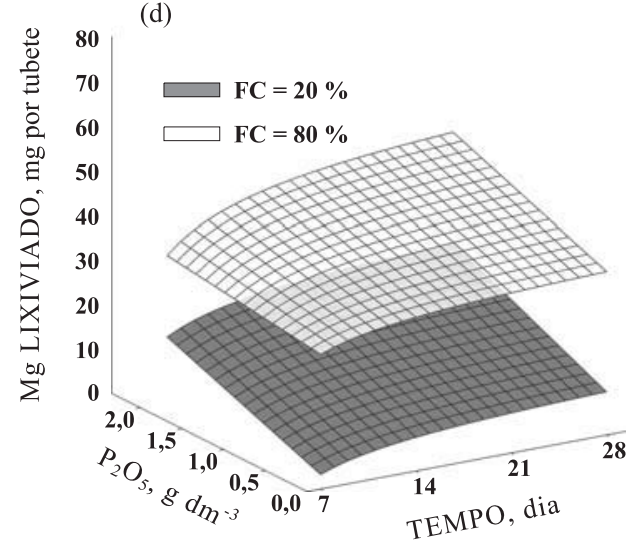

Figura 5. Lixiviação acumulada de $\mathrm{P}$ (a), K (b), Ca (c) e $\mathrm{Mg}$ (d) em função do tempo e da dose de $\mathrm{P}$, para os substratos com 20 e $80 \%$ de fibra de coco (FC). 
favoreceu a sua própria lixiviação e, por reação de troca, a dos demais cátions ( $\mathrm{K}$ e Mg). Como esperado, a lixiviação total do $\mathrm{P}$ foi proporcional à dose aplicada, sendo essa perda mais expressiva, quanto maior a proporção de FC no substrato e o tempo decorrido (Figura 5a), provavelmente devido às interações significativas entre $\mathrm{P}$ e as demais variáveis independentes, substrato e tempo (Quadro 3).

A lixiviação dos cátions foi influenciada pelo substrato (Quadro 3), quanto maior a porcentagem de FC no substrato menor foi a lixiviação de Ca e Mg (Figura 5c,d, respectivamente), certamente porque esse substrato é muito pobre nesses nutrientes (Quadro 1). Para o K, o conteúdo no lixiviado foi alterado significativamente pelo substrato (Quadro 3), sendo o contrário do observado para Ca e Mg, ou seja, quanto maior foi porcentagem de FC, maior foi a perda de $\mathrm{K}$ (Figura 5b), resultado que pode ser explicado pelo mesmo motivo anterior, ou seja, a riqueza do substrato em K (Quadro 1).

A lixiviação de $\mathrm{P}$ foi inversamente proporcional à capacidade de adsorção de $\mathrm{P}$ pelo substrato, inferida pelo P-rem (Quadro 1), ou seja, quanto mais rico em FC era o substrato, menor era sua capacidade de adsorção de $\mathrm{P}$ e maior foi a lixiviação de $\mathrm{P}$ (Figura 5a). Essa relação inversa entre adsorção e lixiviação de $\mathrm{P}$ em substratos também foi constatada por Marconi \& Nelson (1984) e Williams \& Nelson (1996).

\section{CONCLUSÕES}

1. Na fase de enraizamento de estacas de cacau, submetidas à irrigação intermitente com alta freqüência, foi alta a lixiviação de nutrientes, principalmente nos primeiros dias.

2. A perda de $\mathrm{P}$ por lixiviação foi diretamente proporcional à dose aplicada, sendo intensificada quanto menor a capacidade do substrato em adsorver fosfato.

3. A lixiviação dos cátions básicos foi proporcional à riqueza do nutriente no substrato, sendo aumentada com a adição de superfosfato triplo.

\section{LITERATURA CITADA}

ANDRADE, A.T.; FERNANDES, L.A. \& FAQUIN, V. Organic residue, limestone, gypsum and phosphorus adsorption by loads soils. Sci. Agric., 59:349-355, 2002.

ALVAREZ V., V.H.; NOVAIS, R.F.; DIAS, L.E. \& OLIVEIRA, J.A. Determinação e uso do fósforo remanescente. B. Inf. SBCS, 25:27-32, 2000.

BATAGLIA, O.C. \& FURLANI, P.R. Nutrição mineral e adubação para cultivos em substratos com atividade química. In: ENCONTRO NACIONAL SOBRE SUBSTRATOS PARA PLANTAS, 4., Viçosa, MG, 2004. Anais. Viçosa, MG, Universidade Federal de Viçosa, 2004. p.106-128.
BATAGLIA, O.C.; QUAGgio, J.A.; ABREU, M.F. \& BOAVENTURA, P.S.R. Nutrient uptake and leaching on citrus nursery production in substrate with two fertilizer management programs. Acta Hortic., 697:281284, 2005 .

BIRRENKOTT, B.A.; CRAIG, J.L. \& MCVEY, G.R. Effects of substrate type on plant growth and nitrate leaching in cut flower production of oriental lily. HortScience, 40:21352137, 2005.

BRASIL. Leis e Decretos etc. Poder Executivo. Instrução Normativa n.46, de 12 de setembro de 2006. Métodos analíticos oficiais para análises de substratos para plantas e condicionadores de solo. Diário Oficial da República Federativa do Brasil, Brasília, 14 de set. de 2006. n.177, seção 1, p.2-3.

BROSCHAT, T.K. Nitrate, phosphate, and potassium leaching from container-grown plants fertilized by several methods. HortScience, 30:74-77, 1995.

EVANS, H. Investigations on the propagation of cacao. Tropical. Agric., 28:147-203, 1951.

GODOY, A. \& COLE, J.C. Phosphorus source affects phosphorus leaching and growth of containerized Spirea. HortScience, 35:1249-1252, 2000.

KERR, G.P \& HANAN, J.J. Leaching of container media. J. Am. Soc. Hortic. Sci., 110:474-480, 1985.

KU, C.S.M. \& HERSHEY, D.R. Growth response, nutrient leaching, and mass balance for potted poinsettia. II. Phosphorus. J. Am. Soc. Hortic. Sci., 122:459-464, 1997.

MARCONI, D.J. \& NELSON, P.V. Leaching of applied phosphorus in container media. Sci. Hortic., 22:275-285, 1984.

MURRAY, D.B. A new technique in the vegetative propagation of cacao. Rep. Cacao Res., 1953: 53-55, 1954.

PYKE, E.E. The vegetative propagation of Theobroma cacao by softwood cuttings. Tropical Agric., 8:249, 1931.

RAMOS, A.; SOUZA JR., J.O.; MARROCOS, P.C.L.; SODRÉ, G.A.; BRITO, A.M.L.; OLIVEIRA, G.S. \& PACRECO JR., R.G. Sobrevivência e enraizamento de mudas de cacau clonadas em diferentes regimes hídricos. In: ENCONTRO NACIONAL SOBRE SUBSTRATOS PARA PLANTAS, 5., Ilhéus, 2006. Anais. Ilhéus, Comissão Executiva do Plano da Lavoura Cacaueira, 2006. p.165.

SACRAMENTO, C.K.; FARIA, J.C.; PALACIOS, J.B. \& CERQUEIRA, L.S. Enraizamento e crescimento de estacas de cacaueiro (clone TSH 1188) em função do tempo de estaqueamento. In: CONGRESSO BRASILEIRO DE FISIOLOGIA VEGETAL, 8., Ilhéus, 2001. Anais. Ilhéus, Sociedade Brasileira de Fisiologia Vegetal, 2001. CDROM.

SIQUEIRA, P.R. \& ALMEIDA, H.A. Uso de um dispositivo "lisimétrico" para medida do balanço hídrico em mudas clonais de cacau cultivadas em tubetes. In: INTERNATIONAL COCOA RESEARCH CONFERENCE, 14., Accra, 2003. Proceedings. Sabah, Cocoa Producers' Alliance, 2005. p.487-492. 
SONNEVELD, C.; van der ENDE, J. \& van DIJK, P.A. Analysis of growing media by means of a 1:11/2 volume extract. Comm. Soil Sci. Plant Anal., 5:183-202, 1974.

THOMAS, S. \& PERRY JR., F.B. Ammonium nitrogen accumulation and leaching from an all pine bark medium. Hort. Science, 15:824-824, 1980.

VERHAGEN, J.B.G.M. CEC and the saturation of the adsorption complex of coir dust. Acta Hortic., 481:151$155,1999$.
WILLIAMS, K.A. \& NELSON, P.V. Modifying a soilless root medium with aluminum influences phosphorus retention and Chrysanthemum growth. Hort. Science, 31:381-384, 1996.

YEAGER, T.H. \& BARRETT, J.E. Phosphorus leaching from ${ }^{32} \mathrm{P}$-superphosphate-amended soilless container media. Hort. Science, 19:216-217, 1984.

YEAGER, T.H. \& WRIGHT, R.D. Pine bark - Phosphorus relationships. Comm. Soil Sci. Plant Anal., 13:57-66, 1982. 